\title{
FOLFOX-6 Regimen
}

National Cancer Institute

\section{Source}

National Cancer Institute. FOLFOX-6 Regimen. NCI Thesaurus. Code C63591.

A regimen consisting of leucovorin, infusional fluorouracil and oxaliplatin used for the treatment of advanced-stage and metastatic colorectal cancer. This particular version differs from the original FOLFOX (FOLFOX4) regimen with regards to both the doses and the administration of the three-drug combination. An alternative version, referred to as modified-FOLFOX6, is also used in clinical practice. 